\title{
Perfil químico da farinha extrusada de sorgo do genótipo BRS 305 por paper spray ${ }^{1}$
}

\author{
Chemical profile of extruded sorghum flour the genotype BRS 305 by paper spray \\ Perfil químico de harina de sorgo extruido del genotipo BRS 305 con paper spray
}

Recebido: 21/12/2020 | Revisado: 21/12/2020 | Aceito: 17/01/2021 | Publicado: 21/01/2021

\author{
Vinícius Tadeu da Veiga Correia \\ ORCID: https://orcid.org/0000-0001-9789-6015 \\ Universidade Federal de Minas Gerais, Brasil \\ E-mail: viniciustadeu18@hotmail.com \\ Danielle Fátima D'Angelis \\ ORCID: https://orcid.org/0000-0002-5561-4638 \\ Universidade Federal de Minas Gerais, Brasil \\ E-mail: danielledangelis71@ hotmail.com \\ Maria Clara Coutinho Macedo \\ ORCID: https://orcid.org/0000-0001-6769-4623 \\ Universidade Federal de Minas Gerais, Brasil \\ E-mail: clara.macedosl@hotmail.com \\ Ana Luiza Coeli Cruz Ramos \\ ORCID: https://orcid.org/0000-0001-5449-0154 \\ Universidade Federal de Minas Gerais, Brasil \\ E-mail: analuizacoeli@gmail.com \\ Ana Luiza Santos Vieira \\ ORCID: https://orcid.org/0000-0002-3757-6009 \\ Universidade Federal de Minas Gerais, Brasil \\ E-mail: anavieiranutricionista@gmail.com \\ Valéria Aparecida Vieira Queiroz \\ ORCID: https://orcid.org/0000-0002-0156-9827 \\ Embrapa Milho e Sorgo, Brasil \\ E-mail: valeria.vieira@embrapa.br \\ Rodinei Augusti \\ ORCID: https://orcid.org/0000-0002-9448-9518 \\ Universidade Federal de Minas Gerais, Brasil \\ E-mail: augusti.rodinei@gmail.com \\ Andreza Angélica Ferreira \\ ORCID: https://orcid.org/0000-0002-3152-9113 \\ Universidade Federal de Minas Gerais, Brasil \\ E-mail: andrezaferreira@ufmg.br \\ Camila Argenta Fante \\ ORCID: https://orcid.org/0000-0002-1707-3850 \\ Universidade Federal de Minas Gerais, Brasil \\ E-mail: camilafante@ufmg.br \\ Júlio Onésio Ferreira Melo \\ ORCID: https://orcid.org/0000-0002-7483-0942 \\ Universidade Federal de São João del-Rei, Brasil \\ E-mail: onesiomelo@gmail.com
}

\begin{abstract}
Resumo
O objetivo deste trabalho foi determinar os constituintes químicos da farinha extrusada de sorgo (FES) do genótipo BRS 305 empregando-se a espectrometria de massas com ionização por paper spray (PS/MS), um método que apresenta alta precisão, sensibilidade e possui baixo custo de operação. Foram preparados extratos metanólicos a partir da amostra de farinha extrusada de sorgo e posteriormente avaliados nos modos de ionização positivo e negativo. No modo negativo de ionização foi possível identificar 21 compostos, compreendendo as classes dos flavonoides $(48,0 \%)$, fenilpropanoides $(28,0 \%)$, ácidos orgânicos $(9,0 \%)$, taninos condensados, ácidos graxos e carboidratos (5,0\% cada). Adicionalmente no modo de ionização positivo foi possível identificar 9 compostos, pertencentes às classes dos flavonoides (34,0\%), carboidratos (22,0\%), aminoácidos (22,0\%), ácidos graxos e terpenoides (11,0\% cada). A espectrometria de massas por paper spray mostrou-se uma técnica eficiente para rápida
\end{abstract}

\footnotetext{
${ }^{1}$ Trabalho apresentado no CBCP 2020 - Congresso on-line Brasileiro de Tecnologia de Cereais e Panificação, selecionado para publicação na forma de artigo completo.
} 
obtenção de finger prints da amostra de FES avaliada, sendo capaz de identificar um total de trinta compostos químicos, reforçando as propriedades nutricionais e bioativas atribuídas à alguns genótipos de sorgo como o BRS 305 . Palavras-chave: Cereal; Sorghum bicolor; PS/MS.

\begin{abstract}
The objective of this study was to determine the chemical constituents of extruded sorghum flour (ESF) of genotype BRS 305 using mass spectrometry with paper spray ionization (PS/MS), a method that presents high precision, sensitivity and has low operating cost. Methanolextracts were prepared from the extruded sorghum flour sample and subsequently evaluated in positive and negative ionization modes. In the negative mode of ionization it was possible to identify 21 compounds, comprising the classes of flavonoids $(48.0 \%)$, phenylpropanoids $(28.0 \%)$, organic acids $(9.0 \%)$, condensed tannins, fatty acids and carbohydrates (5.0\% each). Additionally in the positive ionization mode, it was possible to identify 9 compounds, belonging to the classes of flavonoids (34.0\%), carbohydrates $(22.0 \%)$, amino acids $(22.0 \%)$, fatty acids and terpenoids (11.0\% each). The mass spectrometry by paper spray proved to be an efficient technique for fast finger prints of the (ESF) sample evaluated, identifying a total of thirty chemical compounds, reinforcing the nutritional and bioactive properties attributed to some sorb genotypes such as BRS 305 .
\end{abstract}

Keywords: Cereal; Sorghum bicolor; PS/MS.

\title{
Resumen
}

El objetivo de este trabajo fue determinar los constituyentes químicos de la harina de sorgo extruido (HSE) del genotipo BRS 305 mediante espectrometría de masas con ionización por paper spray (PS/MS), método que presenta alta precisión, sensibilidad y baja costo de operación. Se prepararon extractos metanólicos a partir de la muestra de harina de sorgo extruida y posteriormente se evaluaron en modos de ionización positiva y negativa. En el modo de ionización negativa fue posible identificar 21 compuestos, comprendiendo las clases de flavonoides (48,0\%), fenilpropanoides $(28,0 \%)$, ácidos orgánicos $(9,0 \%)$, taninos condensados, ácidos grasos y carbohidratos $(5,0 \%$ cada uno). Adicionalmente en el modo de ionización positiva fue posible identificar 9 compuestos, pertenecientes a las clases de flavonoides (34.0\%), carbohidratos (22.0\%), aminoácidos $(22.0 \%)$, ácidos grasos y terpenoides (11.0\% cada uno). La espectrometría de masas por paper spray demostró ser una técnica eficiente para la obtención rápida y eficiente de huellas dactilares de la muestra (HSE) evaluada, pudiendo identificar un total de treinta compuestos químicos, reforzando las propiedades nutricionales y bioactivas atribuidas a algunos genotipos de sorgo como el BRS 305.

Palabras clave: Cereal; Sorghum bicolor; PS/MS.

\section{Introdução}

O sorgo é um cereal sem glúten comumente consumido pela população de países africanos e asiáticos em desenvolvimento (Dicko et al., 2006). No Brasil, a sua utilização na alimentação humana ainda é incipiente, mas nos últimos anos vem ganhando destaque em pesquisas científicas, devido às suas características nutricionais como maior concentração de compostos fenólicos entre os cereais (Pontieri et al., 2013), alto teor de amido resistente em determinados genótipos (Teixeira et al., 2016) e em termos agronômicos, já que apresenta baixo custo de produção e tolerância ao déficit hídrico (Albuquerque et al., 2010).

Dentre os genótipos de sorgo existentes, o BRS 305 é caracterizado por possuir pericarpo marrom, concentrações relevantes de taninos condensados e endosperma mais farináceo (Queiroz et al., 2015). Em termos físico-químicos, a farinha extrusada proveniente destes grãos, apresenta teor de umidade acima de $8 \%$, com concentrações de proteínas, lipídeos, cinzas e carboidratos iguais a 12,48\%,1,72\%,1,42\% e 76,17\%, respectivamente (Correia et al., 2020).

Para avaliação do perfil químico do sorgo, algumas técnicas já foram utilizadas, incluindo cromatografia líquida com espalhamento evaporativo da luz, eletroforese em gel de poliacrilaminada e até mesmo a cromatografia gasosa acoplada à espectrometria de massas. Entretanto, todas essas metodologias requerem extensa preparação laboratorial e altos custos operacionais, o que dificulta a execução das avaliações (Campelo et al., 2020).

Neste cenário, a espectrometria de massas com ionização em paper spray tem sido utilizada na avaliação e controle de qualidade de várias matrizes alimentares de origem vegetal, tais como grumixama (Ramos et al., 2020), açaí (Minighin et al., 2020), gengibre (Oliveira et al., 2020), azeite de oliva (Mazzotti et al., 2013), cagaita (Silva et al., 2019), farinha de banana (Silva et al., 2020), cacau (Oliveira-Júnior et al., 2020), nêspera (Silva et al., 2020b), chá (Deng \& Yang, 2013), café (Garrett 
et al., 2013) e pera-do-cerrado (Mariano et al., 2020), por apresentar alta precisão, sensibilidade e possuir baixo custo de operação.

Diante do exposto, o objetivo deste trabalho foi determinar os constituintes químicos da farinha extrusada de sorgo do genótipo BRS 305 empregando-se a espectrometria de massas com ionização por paper spray nos modos positivo e negativo.

\section{Metodologia}

O presente estudo trata-se de uma pesquisa laboratorial com natureza quali-quantitativa (Pereira et al., 2018).

\subsection{Farinha extrusada de sorgo}

Este trabalho foi realizado no Departamento de Química da Universidade Federal de Minas Gerais. Os grãos de sorgo do genótipo BRS 305, com pericarpo marrom, tanino e testa pigmentada (safra 2016/2017) foram fornecidos pela Embrapa Milho e Sorgo (Sete Lagoas, Minas Gerais) e a farinha extrusada foi processada na Embrapa Agroindústria de Alimentos, Rio de Janeiro, em extrusora dupla-rosca, modelo EV 025 (Clextral, Firminy, França) com matriz circular de quatro furos de mesmo diâmetro (3,8 mm) (Vargas-Solòrzano et al., 2014).

\subsection{Preparação do extrato de farinha de sorgo}

A extração foi realizada de acordo com os procedimentos descritos por Rufino et al. (2010). Inicialmente, a farinha extrusada de sorgo $(2,5 \mathrm{~g})$ foi misturada com $10 \mathrm{~mL}$ de metanol/água $(50: 50 \mathrm{v} / \mathrm{v})$ e a solução foi mantida em repouso por $1 \mathrm{~h}$ em temperatura ambiente $\left(25 \pm 2{ }^{\circ} \mathrm{C}\right)$. Em seguida, as amostras foram centrifugadas a $3493 \times g$ por 22 min $($ Excelsa II, FANEM, 206BL, Brasil) e o sobrenadante recuperado. Posteriormente, $10 \mathrm{~mL}$ de acetona/água (70:30, v/v) foram adicionados ao resíduo, e uma nova centrifugação foi realizada, repetidas as mesmas condições. Ao final, ambos os sobrenadantes foram misturados e acrescidos de água destilada até que $25 \mathrm{~mL}$ fossem alcançados.

\subsection{Perfil químico}

O perfil químico foi avaliado em espectrômetro de massas LCQ Fleet (Thermo Scientific, San Jose, CA, EUA) equipado com fonte de ionização por paper spray. A análise foi executada nos modos positivo e negativo de ionização, onde $2,0 \mu \mathrm{L}$ do extrato e $40 \mu \mathrm{L}$ de metanol foram aplicados em papel cromatográfico, cortado em formato triangular (equilátero $1,5 \mathrm{~cm}$ ) e posicionado à frente da entrada do equipamento.

As condições instrumentais de análise foram: tensão da fonte PS-MS igual a $-3,0 \mathrm{kV}$ (modo negativo) e $+4,0 \mathrm{kV}$ (modo positivo), tensão capilar de $40 \mathrm{~V}$, voltagem de lentes de tubo de $120 \mathrm{~V}$, temperatura do tubo de transferência de $275^{\circ} \mathrm{C}$ e faixa de massa de 100 a 1000. Para identificação dos compostos em análise foi realizada uma comparação entre as razões massa/carga obtidas no estudo com as encontradas na literatura, mediante fragmentação por espectrometria de massa sequencial. A energia de colisão utilizada para fragmentar os compostos variou de 15 a $40 \mathrm{~V}$ e os espectros de massas obtidos foram processados no software Xcalibur (Silva et al., 2019; Ramos et al., 2020).

\section{Resultados e Discussão}

A Figura 1 mostra o espectro gerado a partir da amostra de farinha extrusada de sorgo no modo negativo de ionização. 
Figura 1. Espectrograma PS-MS no modo de ionização negativo de uma amostra de farinha extrusada de sorgo do genótipo BRS 305.

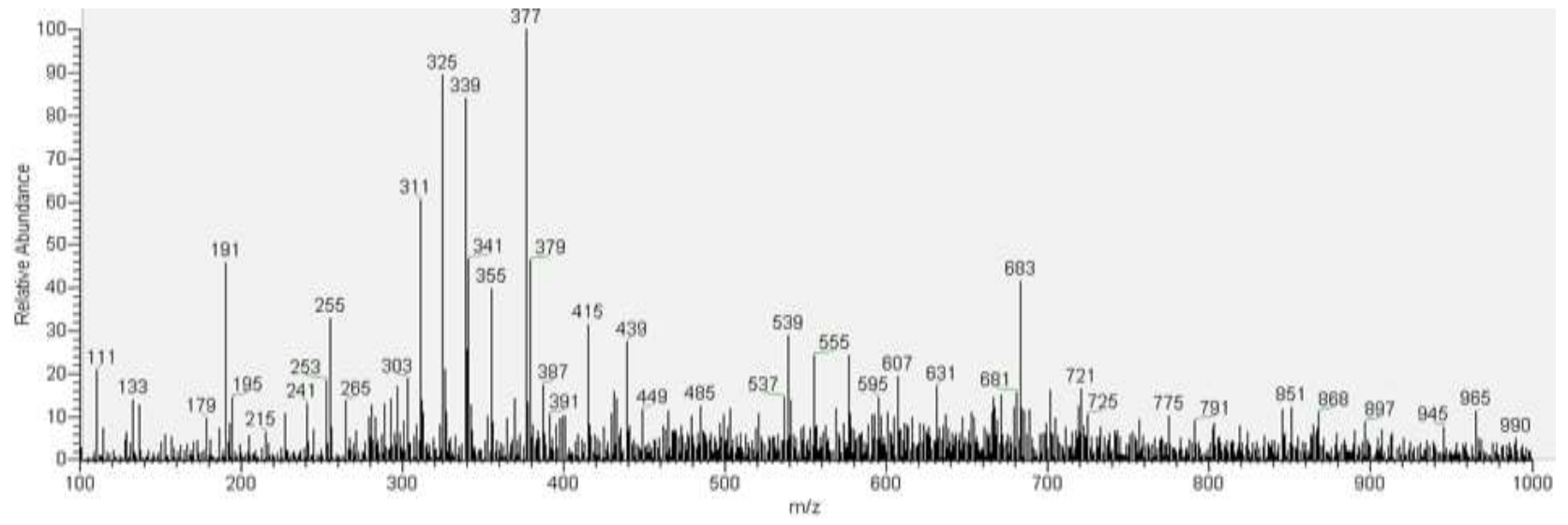

Fonte: Autores (2020).

Todas as identificações no modo de ionização negativo estão demonstradas na Tabela 1.

Tabela 1. Perfil químico da amostra de farinha extrusada de sorgo do genótipo BRS 305 por PS-MS em modo negativo.

\begin{tabular}{|c|c|c|c|c|}
\hline $\mathbf{N}^{\circ}$ & Tentativa de identificação & $\mathbf{m} / \mathbf{z}$ & MS/MS & Referência \\
\hline \multicolumn{5}{|c|}{ Flavonoides } \\
\hline 1 & Catequina & 289 & 205,245 & (Kajdžanoska et al., 2010) \\
\hline 2 & Taxifolina & 303 & 125 & (Chen et al., 2016) \\
\hline 3 & Desoxiclovamida & 326 & 282 & (Cádiz-Guerrea, 2014) \\
\hline 4 & $\begin{array}{l}\text { Apigenina-6-C-glucosídeo } \\
\text { (isovitexina) }\end{array}$ & 431 & 269,341 & (Kang et al., 2016) \\
\hline 5 & $\begin{array}{l}\text { Naringenina } \\
\text { (Hexosídeo) }\end{array}$ & 433 & $271,313,415$ & $\begin{array}{c}\text { (Campelo et al., 2020; Kang et } \\
\text { al., 2016) }\end{array}$ \\
\hline 6 & $\begin{array}{l}\text { Diidrokaempferol } \\
\text { (Hexosídeo) }\end{array}$ & 449 & $\begin{array}{c}269,287,329, \\
405\end{array}$ & $\begin{array}{c}\text { (Campelo et al., 2020; Kang et } \\
\text { al., 2016) }\end{array}$ \\
\hline 7 & $\begin{array}{l}\text { Taxifolina } \\
\text { (Hexosídeo) }\end{array}$ & 465 & $\begin{array}{c}259,285,303, \\
447\end{array}$ & $\begin{array}{c}\text { (Campelo et al., 2020; Kang et } \\
\text { al., 2016) }\end{array}$ \\
\hline 8 & $\begin{array}{c}\text { (Epi) catequina- (Epi) } \\
\text { catequina (procianidina B IV) }\end{array}$ & 577 & $\begin{array}{c}451,425,407 \\
289,287\end{array}$ & (Campelo et al., 2020) \\
\hline 9 & Kaempferol-3-O-rutinoside & 593 & 285 & (Silva et al., 2020) \\
\hline
\end{tabular}


10

Quercetina-3-O-

arabinoglicosídeo
595
(Iswaldi et al., 2011; Campelo et

al., 2020)

\begin{tabular}{|c|c|c|c|c|}
\hline \multicolumn{5}{|c|}{ Taninos condensados } \\
\hline 11 & Proantocianidina & 591 & 289 & (Cádiz-Gurrea, et al.,2014) \\
\hline \multicolumn{5}{|c|}{ Fenilpropanoides } \\
\hline 12 & Ácido cafeico & 179 & 135 & (Campelo et al., 2020) \\
\hline 13 & $\begin{array}{l}\text { Ácido } p \text {-cumárico } \\
\text { (Hexosídeo) }\end{array}$ & 325 & 187 & (Kajdžanoska et al., 2010) \\
\hline 14 & 1,3-O- dicafeoiglicerol & 415 & $\begin{array}{c}135,161,179, \\
253\end{array}$ & (Campelo et al., 2020) \\
\hline 15 & Ácido rosmarínico sulfatado & 439 & 439 & (Barros et al., 2013). \\
\hline 16 & $\begin{array}{l}\text { Ácido cafeoil-cumarico- } \\
\text { quínico }\end{array}$ & 499 & 337 & $\begin{array}{l}\text { (Kang et al., 2016; Silva et al., } \\
\text { 2020b). }\end{array}$ \\
\hline 17 & $\begin{array}{c}\text { Ácido litospérmico A } \\
\text { (isômero) }\end{array}$ & 537 & $269,313,493$ & (Barros et al., 2013) \\
\hline
\end{tabular}

\section{Ácidos orgânicos}

\begin{tabular}{|c|c|c|c|c|}
\hline 18 & Ácido málico & 133 & 115 & $\begin{array}{c}\text { (Silva et al., 2020; Kang et al., } \\
\text { 2016) }\end{array}$ \\
\hline 19 & Ácido cítrico & 191 & 173,111 & $\begin{array}{c}\text { (Wang et al., 2017; Ramos et } \\
\text { al., 2020) }\end{array}$ \\
\hline \multicolumn{5}{|c|}{ Ácido graxo } \\
\hline 20 & Ácido palmítico & 255 & 237 & $\begin{array}{c}\text { (Wang et al., 2017; Ramos et } \\
\text { al., 2020) }\end{array}$ \\
\hline \multicolumn{5}{|c|}{ Carboidrato } \\
\hline 21 & Sacarose & 377 & 341 & $\begin{array}{c}\text { (Silva et al., 2019; Campelo et } \\
\text { al., 2020). }\end{array}$ \\
\hline
\end{tabular}

Fonte: Autores (2020).

A identificação dos compostos no modo de ionização negativo distinguiu entre flavonoides, fenilpropanoides, ácidos orgânicos, ácido graxo, carboidrato e taninos condensados. As proporções das classes químicas encontradas estão demonstradas na Figura 2. 
Figura 2. Proporção de classes químicas encontradas na análise do modo de ionização negativa por PS/MS.

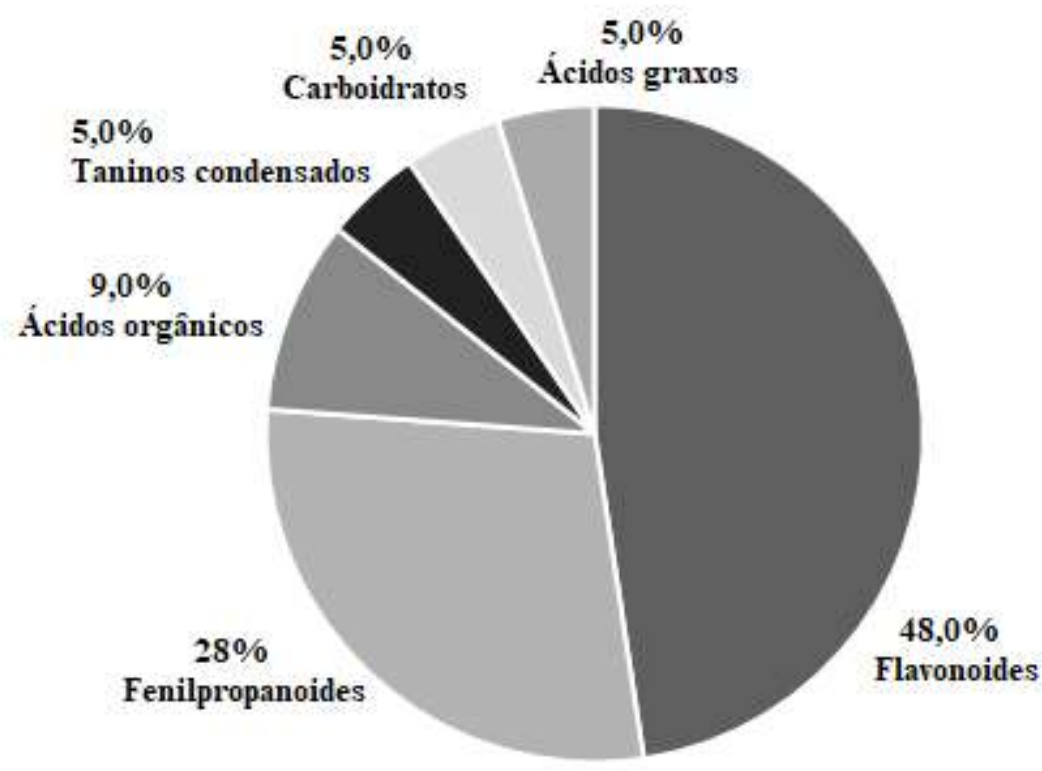

Fonte: Autores (2020).

Os flavonoides são metabólitos secundários de plantas e o maior grupo de fenólicos naturais presentes em vegetais. Em geral, dietas alimentares ricas nestes compostos são associadas a ação preventiva de doenças crônicas não-transmissíveis, com potencial antioxidante, antiinflamatório, anticarcinogênico e capacidade de proteção cardiovascular (Awika \& Rooney, 2004; Moraes et al., 2012; Queiroz et al., 2018). São divididos nas principais subclasses: flavonóis, flavonas, flavanonas, flavanas, isoflavonoides e antocianinas (Beecher, 2003).

A flavona apigenina foi identificada pelo íon $\mathrm{m} / \mathrm{z} 431$, enquanto a flavanona naringenina pelo íon $\mathrm{m} / \mathrm{z} 433$. Estes compostos também foram encontrados em sorgos com pericarpo marrom, vermelho, amarelo, branco e preto nos trabalhos de Dykes et al. (2011), Yang et al. (2012) e Cardoso et al. (2015) ao avaliarem amostras de sorgo por cromatografia líquida de alta eficiência.

De acordo com Campelo et al. (2020), o hexosídeo de taxifolina (m/z 465) é indicado como o polifenol mais abundante em alguns genótipos de sorgo, na subclasse flavan-3-ol destacam-se os compostos catequina (m/z 289), (Epi) catequina- (Epi) catequina (procianidina B IV) (m/z 577), moléculas que representam uma grande proporção de compostos fenólicos totais deste cereal (Devil et al., 2012).

No presente estudo, os fenilpropanoides que predominaram na amostra de farinha extrusada de sorgo do genótipo BRS 305 foram o ácido cafeico (m/z 179), ácido $p$-cumárico (m/z 325), ácido rosmarínico sulfatado (m/z 439), ácido cafeoilcumarico-quínico (m/z 499), ácido litospérmico A (isômero) (m/z 537) e 1,3-O- dicafeoiglicerol (m/z 415). Quanto a fração lipídica em farinhas de sorgo, esta representa em torno de $3 \%$ de sua composição nutricional, estando presente nas concentrações dos principais ácidos graxos, como ácido oleico (31,1 a 48,9\%), ácido linoleico (27,6 a 50,7\%) e palmítico (11,7 a 20,2\%), sendo este último identificado no trabalho pelo íon m/z 255 (Queiroz et al., 2011; Martino et al., 2012). Em relação aos ácidos orgânicos, prevaleceram os ácidos cítrico (m/z 191) e málico (m/z 133), também encontrados no trabalho de Silva et al. (2020) ao analisarem farinhas elaboradas da casca de banana.

As proantocianidinas (m/z 591), também conhecidas como taninos condensados estão entre os mais importantes antioxidantes conhecidos (Liu et al., 2013). Na cultura do sorgo, estes compostos apresentam um grande papel agronômico, ao reduzir a predação de aves e danos ao grão pré e pós-colheita (Kaufman et al., 2012). Quanto as características funcionais e 
nutricionais, estes nutrientes estão associados a benefícios à saúde em função de sua ação antioxidante, atividade cardioprotetora e efeito antitromóticos. Ainda, podem auxiliar na redução da disponibilidade calórica do alimento ao complexar com macronutrientes, como proteínas e carboidratos (Moraes et al., 2012; Wu et al., 2012).

Quanto ao modo de ionização positivo, foram identificados nove compostos distintos compreendendo as classes químicas dos carboidratos, aminoácidos, flavonoides, ácidos graxos e terpenoides. O espectro PS (+) - MS da análise de farinha extrusada de sorgo está mostrado na Figura 3 e as identificações no modo de ionização positiva estão demonstradas na Tabela 2 .

Figura 3. Espectrograma PS-MS no modo de ionização positivo de uma amostra de farinha extrusada de sorgo do genótipo BRS 305.

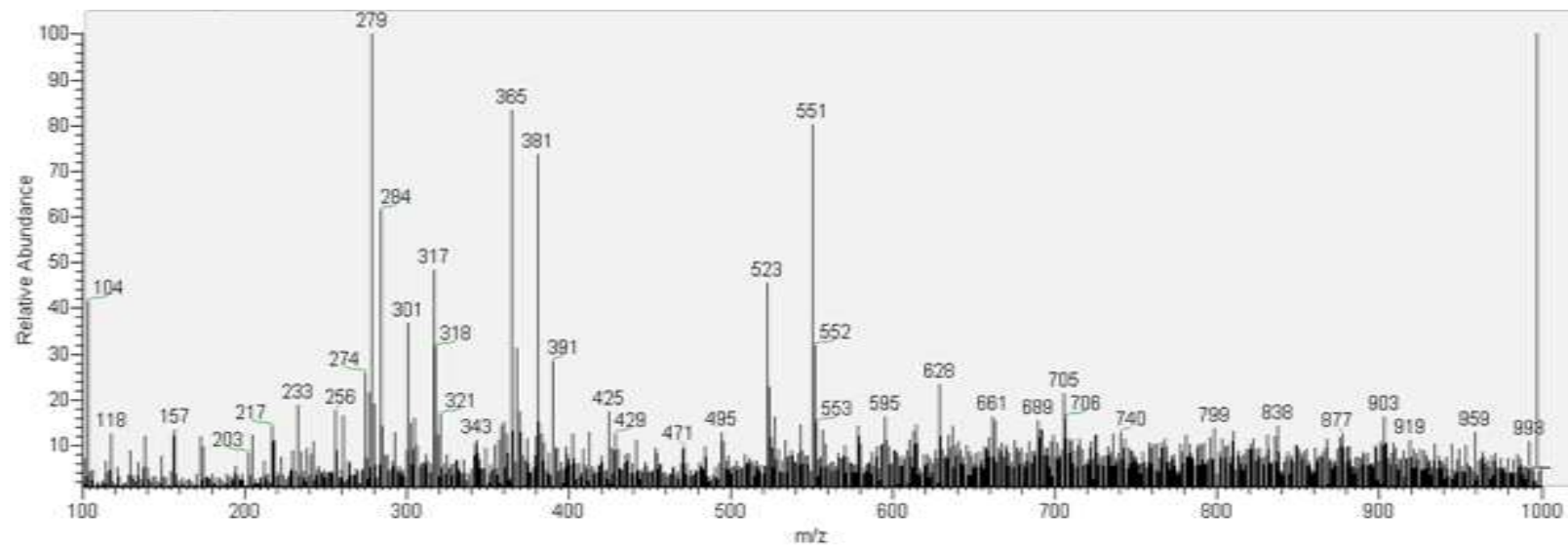

Fonte: Autores (2020). 
Research, Society and Development, v. 10, n. 1, e40710111414, 2021

(CC BY 4.0) | ISSN 2525-3409 | DOI: http://dx.doi.org/10.33448/rsd-v10i1.11414

Tabela 2. Perfil químico da amostra de farinha de sorgo extrusada do genótipo BRS 305 por PS-MS em modo positivo.

\begin{tabular}{|c|c|c|c|c|}
\hline $\mathbf{N}^{\circ}$ & Tentativa de identificação & $\mathbf{m} / \mathbf{z}$ & MS/MS & Referência \\
\hline \multicolumn{5}{|c|}{ Carboidratos } \\
\hline 1 & Glicose & 203 & - & (Guo et al., 2017) \\
\hline 2 & Sacarose & 381 & 219 & (Campelo et al., 2020) \\
\hline \multicolumn{5}{|c|}{ Aminoácidos } \\
\hline 3 & Glicina-betaína & 118 & 59 & (Campelo et al., 2020) \\
\hline 4 & Histidina & 156 & 110 & (Campelo et al., 2020) \\
\hline \multicolumn{5}{|c|}{ Flavonoides } \\
\hline 5 & Miricetina-3-glcA & 495 & 319 & (Ramos et al., 2020) \\
\hline 6 & $\begin{array}{c}\text { Dímero de apigeninidina-7-O- } \\
\text { metilflaveno }\end{array}$ & 523 & - & (Geera et al., 2012) \\
\hline 7 & $\begin{array}{c}6,8 \text {-Di-C- } \beta \text {-glucopiranosil } \\
\text { crisina }\end{array}$ & 579 & 561,525 & (Campelo et al., 2020) \\
\hline \multicolumn{5}{|c|}{ Ácido graxo } \\
\hline 8 & $\begin{array}{c}\text { Éster metílico de cnidiosídeo } \\
\text { B }\end{array}$ & 413 & 413 & (Ren et al., 2016) \\
\hline
\end{tabular}

\section{Terpenoide}

$9 \quad$ Diidroisovaltrato $\quad 425 \quad 281,365 \quad$ Silva et al. (2020)

Fonte: Autores (2020).

As proporções das classes químicas encontradas no modo positivo de ionização estão demonstradas na Figura 4. 
Figura 4. Proporção de classes químicas encontradas na análise do modo de ionização positivo por PS/MS.

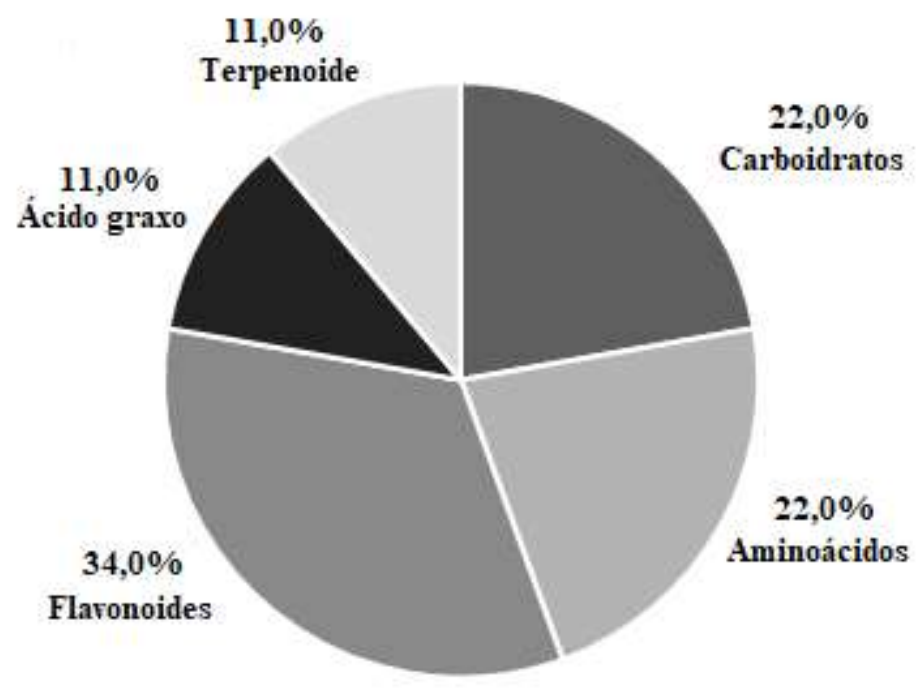

Fonte: Autores (2020).

Em cereais, incluindo o sorgo, os derivados glicosilados de quercetinas, kaempferol e miricetina são as moléculas químicas mais predominantes, destacando assim a presença dos compostos, quercetina-3-O-arabinoglicosídeo m/z 595, kaempferol-3-O-rutinoside m/z 593 (modo negativo) e miricetina-3-glcA m/z 495 (modo positivo) identificados neste trabalho (Przybylska-Balcerek et al., 2019).

De acordo com Vasat (2007), as proteínas e o amido presentes nos grãos de sorgo são digeridos mais vagarosamente, essa condição representa um benefício a pacientes diabéticos, uma vez que a glicose vai sendo liberada aos poucos no organismo. Os açúcares identificados neste trabalho foram a sacarose (m/z 377 negativo e m/z 381 positivo) e a glicose (m/z 203), que, juntamente com o amido, são os carboidratos predominantes em grãos de sorgo. A presença destes compostos em genótipos de sorgo está relacionada ao mecanismo de defesa da planta, associados a alguma situação de estresse (Campelo et al., 2020). Quanto ao conteúdo de aminoácidos foram identificados dois, sendo eles a glicina-betaína (m/z 118) e a histidina $(\mathrm{m} / \mathrm{z} 156)$.

\section{Conclusão}

Carboidratos, aminoácidos, ácidos graxos, ácidos orgânicos e compostos com ação antioxidante, como os flavonoides e os fenilpropanoides representam as principais classes químicas encontradas em FES no modo negativo e positivo, sendo a técnica de espectrometria de massas com ionização por paper spray uma ferramenta interessante para identificar o perfil químico de amostras vegetais.

\section{Referências}

Albuquerque, C. J. B., Rocha, G. R, Brant, R. S. \& Mendes, M. C. (2010). Espaçamento reduzido para o cultivo do sorgo granífero no sistema irrigado e em sequeiro, Pesquisa Aplicada \& Agrotecnologia, Guarapuava, 3, 1-16.

Awika, J. M., \& Rooney, L. W. (2004). Sorghum phytochemicals and their potential impact on human health. Phytochemistry, 65, 1199-1221. DOI: https://doi.org/10.1016/j.phytochem.2004.04.001.

Barros, L., Dueñas, M., Dias, M. I., Sousa, M. J., Santos-Buelga, C., \& Ferreira, I. C. F. R. (2013). Phenolic profiles of cultivated, in vitro cultured and commercial samples of Melissa officinalis L. infusions. Food Chemistry, 136(1), 1-8. https://doi.org/10.1016/j.foodchem.2012.07.107. 
Cádiz-Gurrea, M. L., Lozano-Sanchez, J., Contreras-Gámez, M., Legeai-Mallet, L., Fernández-Arroyo, S. \& Segura-Carretero, A. (2014). Isolation, comprehensive characterization and antioxidant activities of Theobroma cacao extract. ScienceDirect, 10 , 485 - 498 . DOI: http://dx.doi.org/10.1016/j.jff.2014.07.016.

Campelo, F. A., Henriques, G. S., Simeone, M. L. F., Queiroz, V. A. V., Silva, M. R., Augusti, R., Melo, J. O. F., Lacerda, I. C. A. \& Araújo, R. L. B. (2020). Study of thermoplastic extrusion and its impact on the chemical and nutritional characteristics in two sorghum genotypes SC 319 and BRS 332 . Journal of the Brazilian Chemical Society, 31, 788-802. https://doi.org/10.21577/0103-5053.20190243.

Cardoso, L. M., Pinheiro, S. S., Carvalho, C. W. P., Queiroz, V. A. V., Menezes, C. B., Moreira, A. V. B., Barros, F. A. R., Awika, J. M., Martino, H. S. D. \& Sant'ana, H. M. P. (2015). Phenolic compounds profile in sorghum processed by extrusion cooking and dry heat in a conventional oven. Journal of Cereal Science, 65, 220-226. 10.1016/j.jcs.2015.06.015.

Chen, G, Li, X, Saleri, F. \& Guo, M. (2016). Analysis of Flavonoids in Rhamnus davurica and Its Antiproliferative Activities. Molecules, 21(10), 1275. https://doi.org/10.3390/molecules21101275.

Correia, V. T. V., Dangelis, D. F., Rodrigues, C. G., Amante, P. R., Queiroz, V. A. V., Ferreira, A. A. \& Fante, C. A. (2020). Physico-chemical and technological characterization of extruded sorghum flour of the genotype BRS 305. Research, Society and Development, 9, e115984963. http://dx.doi.org/10.33448/rsd-v9i8.4963.

Dicko, M. H., Gruppen, H., Traore, A., Voragen, A. G. J. \& Van Berkel, W. J. H. (2006). Sorghum grain as human food in Africa: relevance of content of starch and amylase activities. African Journal of Biotechnology, 5 (5), 384-395.

Dykes, L., Peterson, G. C., Rooney, W. L. \& Rooney, L. W. (2011). Flavonoid composition of lemon-yellow sorghum genotypes. Food Chemistry, 128 (1), 173- 179. 10.1016/j.foodchem.2011.03.020.

Deng, J., \& Yang, Y. (2013). Chemical fingerprint analysis for quality assessment and control of Bansha herbal tea using paper spray mass spectrometry. Analytica Chimica Acta, 785, 82-90. https://doi.org/10.1016/j.aca.2013.04.056.

Devil, P. S., Saravanakumar, M. \& Mohandas, S. (2012). The effects of temperature and pH on stalility of antocyanins from red sorghum (sorghum bicolor) bran. African Journal of Food Science, 6(24), 567-573.

Garrett, R., Rezende, C. M., \& Ifa, D. R. (2013). Coffee origin discrimination by paper spray mass spectrometry and direct coffee spray analysis. Analytical Methods, 5(21), 5944. https://doi.org/10.1039/c3ay41247d.

Geera, B., Ojwang, L. O. \& Awika, J. M. (2012). New highly stable dimeric 3-deoxyanthocyanidin pigments from sorghum bicolor leaf sheath. Journal of Food Science, 77(5), 566-72. 10.1111/j.1750-3841.2012.02668.x

Guo, Y., Gu, Z., Liu, X., Liu, J., Ma, M., Chen, B. \& Wang, L. (2017). Rapid Analysis of Corni fructus Using Paper Spray-Mass Spectrometry. Phytochemical Analysis, 28(4), 344-350. 10.1002/pca.2681.

Iswaldi, I., Arráez-Román, D., Rodríguez-Medina, I., Beltrán-Debón, R., Joven, J., Segura-Carretero, A. \& Fernández-Gutiérrez, A. (2011). Identification of phenolic compounds in aqueous and ethanolic rooibos extracts (Aspalathus linearis) by HPLC-ESI-MS (TOF/IT). Analytical and Bioanalytical Chemistry, 400(10), 3643-54. 10.1007/s00216-011-4998-z.

Kajdžanoska, M, Gjamovski, V. \& Stefova, M. (2010). HPLC-DAD-ESI-msn identification of phenolic compounds in cultivated strawberries from Macedonia. Macedonian Journal of Chemistry and Chemical Engineering, 29(2), 181-194.

Kang, J., Price, W., Ashton, J. \& Tapsell, L. and Johnson, S. (2016). Identification and characterization of phenolic compounds in hydromethanolic extracts of sorghum wholegrains by LC-ESI-MSn. Food Chemistry, 211, 215-226. 10.1016/j.foodchem.2016.05.052.

Kaufman, R. C., Herald, T. J., Bean, S. R., Wilson, J. D. \& Tuinstra, M. R. (2012). Variability in tannin content, chemistry and activity in a diverse group of tannin containing sorghum cultivars. Journal of the Science of Food and Agriculture, 93(5), 1233-1241. https://doi.org/10.1002/jsfa.5890.

Liu, Y., Feng, S., Song, L., He, G., Chen, M., \& Huang, D. (2013). Secondary Metabolites in Durian Seeds: Oligomeric Proanthocyanidins. Molecules, 18, $14172-14185$.

Mariano, A. P. X., Ramos, A. L. C. C., Augusti, R., Araújo, R. L. B., Melo, J. O. F. (2020). Analysis of the chemical profile of cerrado pear fixed compounds by mass spectrometry with paper spray and volatile ionization by SPME-HS CG-MS. Research, Society and Development, 9, e949998219-22. http://dx.doi.org/10.33448/rsd-v9i9.8219.

Martino, H. S. D., Tomaz, P. A., Moraes, E. A., Conceição, L. L., Oliveira, D. S., Queiroz, V. A. V., Rodrigues, J. A. S., Pirozi, M. R., Pinheiro-Sant'ana, H. M., \& Ribeiro, S. M. R. (2012). Chemical characterization and size distribution of sorghum genotypes for human consumption. Revista Instituto Adolfo Lutz, $71(2), 337-344$

Mazzotti, F., Di Donna, L., Taverna, D., Nardi, M., Aiello, D., Napoli, A., \& Sindona, G. (2013). Evaluation of dialdehydic anti-inflammatory active principles in extra-virgin olive oil by reactive paper spray mass spectrometry. International Journal of Mass Spectrometry, 352, 87-91. https://doi.org/10.1016/j.ijms.2013.07.012.

Minighin, E. C., Anastácio, L. R., Melo, J. O. F. \& Labanca, R. A. (2020). Açai (Euterpe oleracea) e suas contribuições para alcance da ingestão diária aceitável de ácidos graxos essenciais. Research, Society and Development, 9, e760986116. http://dx.doi.org/10.33448/rsd-v9i8.6116.

Moraes, E. A., Natal, D. I. G., Queiroz, V. A. V., Schaffert, R. E., Cecon, P. R., Paula, S. O., Benjamim, L. A., Ribeiro, S. M. R., \& Martino, H. S. D. (2012). Sorghum genotype may reduce low-grade inflammatory response and oxidative stress and maintains jejunum morphology of rats fed a hyperlipidic diet. Food Research International, 49, 553-559. https://doi.org/10.1016/j.foodres.2012.07.029. 
Oliveira, C. T., Ramos, A. L. C. C., Mendonca, H. O. P., Consenza, G. P., Silva, M. R., Fernandes, C., Augusti, R., Melo, J. O. F., Ferreira, A. V. M. \& Araujo, R. L. B. (2020). Quantification of 6-gingerol, metabolomic analysis by paper spray mass spectrometry and determination of antioxidant activity of ginger rhizomes (Zingiber officinale). Research, Society and Development, 9, e366984822. https://doi.org/10.33448/rsd-v9i8.4822.

Oliveira-Júnior, A. H., Ramos, A. L. C. C., Guedes, M. N. S., Fagundes, M. C. P., Augusti, R. \& Melo, J. O. F. (2020). Chemical profile and bioprospecting of cocoa beans analyzed by paper spray mass spectrometry. Research, Society and Development, 9, e975986882. https://doi.org/10.33448/rsd-v9i8.6882.

Pereira, A. S., Shitsuka, D. M., Parreira, F. J., \& Shitsuka, R. (2018). Metodologia da pesquisa científica. UAB/NTE/UFSM. https://repositorio.ufsm .br/bitstream/handle/1/15824/Lic_Computacao_Metodologia-Pesquisa-Cientifica.pdf?sequence=1.

Pontieri, P., Mamone, G., Caro, S., Tuinstra, M. R., Roemer, E., Okot, J., De Vita, P., Ficco, D. B. M., Alifano, P., Pignone, D., Massardo, D. R. \& Del Giudice, L. (2013). Sorghum, a healthy and gluten-free food for celiac patients as demonstrated by genome, biochemical, and immunochemical analyses. Journal of Agricultural and Food Chemistry, 61, 2565-2571. https://doi.org/10.1021/jf304882k.

Przybylska-Balcerek, A., Frankowski, J. \& Stuper-Szablewska, K. (2019). Bioactive compounds in sorghum. European Food Research and Technology, 245(5), 1075-1080. 10.1007/s00217-018-3207-0.

Queiroz, V. A. V., Moraes, E. M., Schaffert, R. E., Moreira, A. V., Ribeiro, S. M. R., \& Martino, H. S. D. (2011). Potencial funcional e tecnologia de processamento do sorgo [Sorghum bicolor (L.) Moench], para alimentação humana. Revista Brasileira de Milho e Sorgo, 10(3), 180-195. https://doi.org/10.18512/1980-6477/rbms.v10n3p180-195.

Queiroz, V. A. V., Silva, C. S, Menezes, C. B., Schaffert, R. E., Guimarães, F. F. M., Guimarães, L. J. M., Guimarães, P. E. O. \& Tardin, F. D. (2015). Nutritional composition of sorghum [Sorghum bicolor (L.) Moench] genotypes cultivated without and with water stress. Journal of Cereal Science, 65, 103111. https://doi.org/10.1016/j.jcs.2015.06.018.

Queiroz, V. A. V., Oliveira, K. G., Paiva, C. L., Carlos, L. A., De Menezes, C. B., Barros, F. A. R., Pinheiro-Sant'ana, H. M. \& Anunciação, P. (2018). Retention of some flavones and flavanones in flour, grain and bran of sorghum during storage. Revista Brasileira de Milho e Sorgo, 17(3), 522-534. https://doi.org/10.18512/1980-6477/rbms.v17n3p522-534.

Ramos, A. L. C. C., Mendes, D. D., Silva, M. R., Augusti, R., Melo, J. O. F., Araújo, R. L. B. \& Lacerda, I. C. A. (2020). Chemical profile of Eugenia brasiliensis (Grumixama) pulp by PS/MS paper spray and SPME-GC / MS solid-phase microextraction. Research, Society and Development, 9 , e318974008. http://dx.doi.org/10.33448/rsd-v9i7.4008.

Ren, Y., Chiang, S., Zhang, W., Wang, X., Lin, Z. \& Ouyang, Z. (2016). Paper-capillary spray for direct mass spectrometry analysis of biofluid samples. Analytical and Bioanalytical Chemistry, 408(5), 1385-1390. 10.1007/s00216-015-9129-9.

Rufino, M. do S. M., Alves, R. E., de Brito, E. S., Pérez-Jiménez, J., Saura-Calixto, F., \& Mancini-Filho, J. (2010). Bioactive compounds and antioxidant capacities of 18 non-traditional tropical fruits from Brazil. Food Chemistry, 121(4), 996-1002. http://dx.doi.org/10.1016/j.foodchem.2010.01.037.

Silva, M., Freitas, L., Souza, A., Araújo, R., Lacerda, I., Pereira, H., Augusti, R. \& Melo, J. (2019). Antioxidant Activity and Metabolomic Analysis of Cagaitas (Eugenia dysenterica) using Paper Spray Mass Spectrometry. Journal of the Brazilian Chemical Society, 30, 1034-1044. https://doi.org/10.21577/0103-5053.20190002.

Silva, V., Arquelau, P., Silva, M., Augusti, R., Melo, J. \& Fante, C. (2020). Use of paper spray-mass spectrometry to determine the chemical profile of ripe banana peel flour and evaluation of its physicochemical and antioxidant properties. Quimica Nova, 43, 579-585. https://doi.org/10.21577/01004042.20170521.

Silva, V. D. M., Macedo, M. C. C., Santos, A. N., Silva, M. R., Augusti, R., Lacerda, I. C. A., Melo, J. O. F. \& Fante, C. A. (2020b). Bioactive activities and chemical profile characterization using paper spray mass spectrometry of extracts of Lindl. leaves. Rapid Communications in Mass Spectrometry, 34 , e8883. https://doi.org/10.1002/rcm.8883.

Teixeira, N. C., Queiroz, V. A. V., Rocha, M. C., Amorim, A. C. P., Soares, T. O., Monteiro, M. A. M., Menezes, C. B., Schaffert, R. E., Garcia, M. A. V. T., \& Junqueira, R. G. (2016). Resistant starch content among several sorghum (Sorghum bicolor) genotypes and the effect of heat treatment on resistant starch retention in two genotypes. Food Chemistry, 197, 291-296. https://doi.org/10.1016/j.foodchem.2015.10.099.

Vargas-Solorzano, J. W., Carvalho, C. W. P., Takeiti, C. Y., Ascheri, J. L. R., \& Queiroz, V. A. V. (2014). Physicochemical properties of expanded extrudates from colored sorghum genotypes. Food Research Internation, 55, 37-44. DOI: https://doi.org/10.1016/j.foodres.2013.10.023.

VASAT: Virtual Academy for the Semi-Arid Tropics. Uses and nutricional features of sorghum.<http://www.icrisat.orglvasatllearning_resources/ crops/sorghum/sorghum_prodpractices/htmllm112/index.html>.

Wang, J, Jia, Z, Zhang, Z, Wang, Y, Liu, X, Wang, L\& Lin, R. (2017). Analysis of Chemical Constituents of Melastoma dodecandrum Lour. by UPLC-ESI-QExactive Focus-MS/MS. Molecules, 22(3), 476. https://doi.org/10.3390/molecules22030476.

Wu, Y., Li, X., Xiang, W., Zhu, C., Lin, Z., Wu, Y., Li, J., Pandravada, S., Ridder, D. D., Bai, G., Wang, M. L., Trick, H. N., Bean, S. R., Tuinstra, M. R., Tesso, T. T. \& Yu, J. (2012). Presence of tannins in sorghum grains is conditioned by different natural alleles of Tannin1. Proceedings of the National Academy of Sciences, 109(26), 10281-10286. https://doi.org/10.1073/pnas.1201700109.

Yang, L., Allred, K. F., Geera, B., Allred, C. D. \& Awika, J. M. (2012). Sorghum phenolics demonstrate estrogenic action and Induce apoptosis in nonmalignant colonocytes. Nutrition and Cancer, 64(3), 419-427. 10.1080/01635581.2012.657333. 